\title{
Analytical study of high absorption region of the absorption edge of a-Si:H using nonlinear regression method
}

\author{
Abdulla I. Abbo', Ekram J. Hassan ${ }^{1}$, Ali Ch. Hussian² \\ ${ }^{1}$ Department of Physics, College of Science for Women, University of Baghdad \\ ${ }^{2}$ Departent of Physics, College of Education, AL-Mustansiriyah University \\ E-mail: ali_hussian14@yahoo.com
}

\begin{abstract}
This research is concerned with the re-analysis of optical data (the imaginary part of the dielectric function $\varepsilon_{2}$ as a function of photon energy E) of a-Si:H films prepared by Jackson et al. and Ferlauto et al. through using nonlinear regression fitting we estimated the optical energy gap $E_{\text {opt }}$ and the deviation from the Tauc model by considering the parameter of energy photon-dependence of the momentum matrix element of the $p$ as a free parameter by assuming that density of states distribution to be a square root function. It is observed for films prepared by Jackson et al. that the value of the parameter $\mathrm{p}$ for the photon energy range $(\sim 2.7-2) \mathrm{eV}$ is 0.787 is close to the value $p=1$ assumed by the Cody model and the optical gap energy is $1.72 \mathrm{eV}$ which is also close to the value of the Cody gap of these films. While for the films prepared by Ferlauto et al. the value of the parameter $p$ is equal to 0.57 and the optical gap energy is $1.72 \mathrm{eV}$.
\end{abstract} Key words

Amorphous silicon, optical properties of silicon, optical data.

Article info.

Received: Dec. 2017 Accepted: Feb. 2018 Published: Jun. 2018

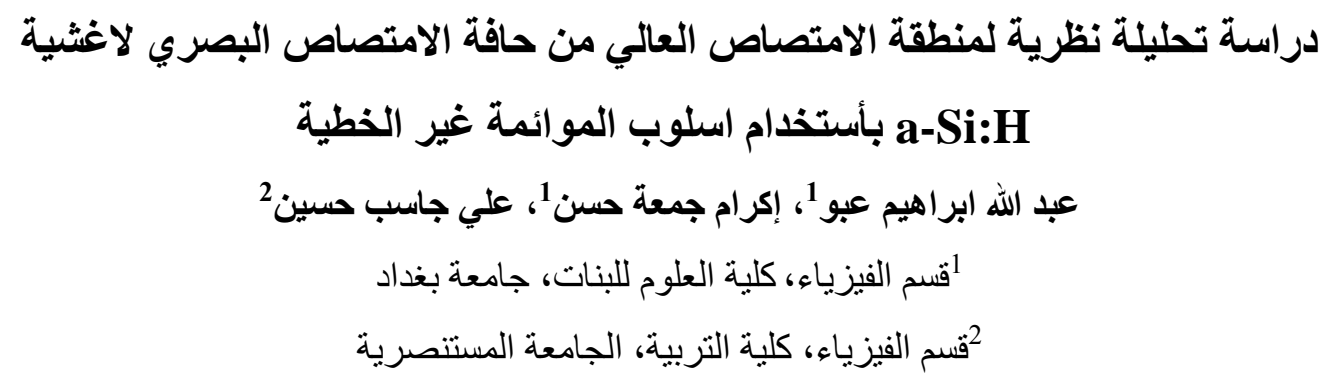

يهتم هذا البحث بأعادة تحليل البيانات البصرية (الجزء الخيالي لدالة العزل بوصفها دالة لطاقة الفونون)

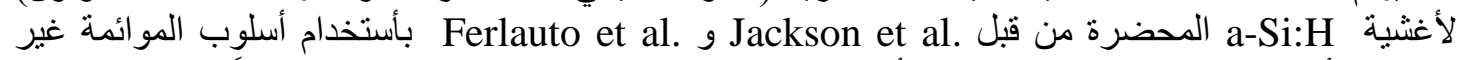

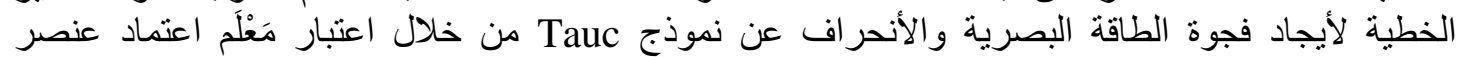

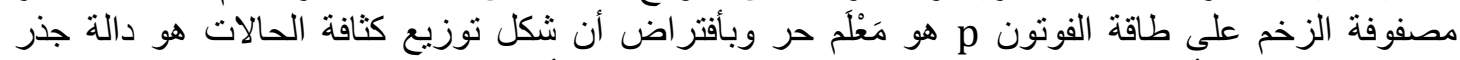

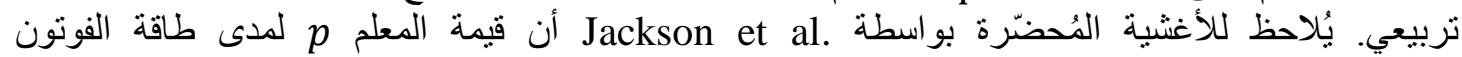

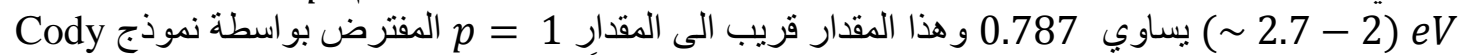
وان قيمة فجوة الطاقة البصرية هي 1.72 eV التي قريبة ايضاً الى قيمة فجوة

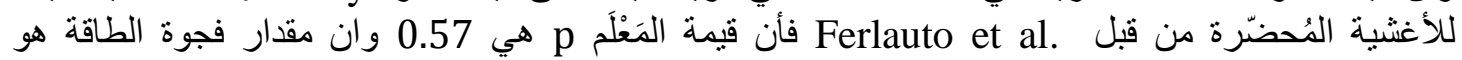

$.1 .72 \mathrm{eV}$

\section{Introduction}

Amorphous silicon (a-Si) remains at the center of attention of modern amorphous/glassy solid state physics community [1]. Although the electronic processors 
(microelectronics) devices is continuously developing with unbelievable miniaturization on the crystalline silicon chip, the input/output devices (large area electronics) has to stay of large dimensions as a necessity of man/machine interface such as document scanners, electronic displays, printers etc... The crystalline silicon could not satisfy the requirement of these large area optoelectronic devices due to its poor optical properties and limited dimensions. The solutions developed on the (amorphous) semiconductors other than the crystalline silicon have created mismatching problems with the existing silicon based microelectronic technologies [2]. In fact, amorphous semiconductors (a-semiconductors) lack long- range order which is the structural characteristic of these materials [3]. In amorphous semiconductors there exist some of the electronic states, referred to as tail states; encroach into the otherwise empty gap region. In addition to tail states, there are other localized states deep within the gap region. These localized tail states in amorphous semiconductors arise as a consequence of imperfect short range order [4]. In addition to the localized tail states, there are bonding defects that may produce midgap states. These bonds are called dangling bonds which affect the optical properties of amorphous semiconductors [5]. Amorphous silicon whose huge amount of dangling bond states at midgap $\left(10^{20} \mathrm{~cm}^{-1} \mathrm{eV}^{-1}\right)$ are reduced to a minute amount $\left(10^{15} \mathrm{~cm}^{-1} \mathrm{eV}^{-1}\right)$ by the hydrogen compensation process, becomes able to be selectively doped both $\mathrm{n}$ and $\mathrm{p}$ types leading to a large number of devices practically useful such as p-n, p-i-n, Schottky diodes etc...[6]. Consequently, the hydrogenated amorphous silicon (a-
$\mathrm{Si}: \mathrm{H})$ seems to be a solution to the large area problem of crystalline silicon because it can be deposited at low temperatures $\left(<300 \mathrm{~K}^{\circ}\right)$ by the glow discharge method at reasonable cost as large area thin films on low cost substrate [7].

The theoretical and applied study of the optical properties of a-Si:H materials is regarded very important and so far has an important focus of research because of the complex nature of the electronic structure compared to their crystalline counterparts with a regular structure, which is easy to study theoretically [8]. The higher absorption region of the optical absorption edge has been selected to study because one of the main parameters derived from the analysis of optical data (the imaginary part of the dielectric function $\varepsilon_{2}$ as a function of photon energy $E$ ) is the optical energy gap which has great interest in theory and practice [8]. Because of the absence of transitional symmetry of the amorphous materials and although there is a short-range symmetry, it is not easy to deal theoretically with the results of the empirical data crucially. Therefore, it is resorted to the modeling by using less rigorous theoretical models than those of crystalline semiconductors (c- semiconductors), such as Tauc and Cody models [9]. A theoretical study of the optical properties of the amorphous silicon films has been carried out by re-analyzing the optical data of the higher absorption region of the optical absorption edge of these films in a nonlinear regression, by considering Density of States (DOS) for both the valance and conduction bands takes the parabolic function and the dependence parameter of the momentum matrix element on photon energy being a free parameter. 


\section{Theoretical part}

The Eq. of the imaginary dielectric function can be written to fit with the data of the high absorption region from the optical absorption edge as follows: [10]

$$
\varepsilon_{2}(E)=K E^{-q}\left(E-E_{O p t}\right)^{r}
$$

where q represents the photon energy dependence coefficient of a square matrix element, $r$ the dependence coefficient of the DOS form on photon energy, $\mathrm{E}_{\text {opt }}$ : A parameter representing the optical energy gap produced by the fitting of empirical data with the Eq. (1). If the plot of $\left(\mathrm{E}^{\mathrm{q}} \varepsilon_{2}\right)^{1 / r}$ as a function of the photon energy $E$ is a straight line, its intersection with the energy axis is the value of the optical energy gap and $\mathrm{K}$ : an experimental constant determined by taking a linestraight slope of power $r$ [10]. Since the plot of the straight line between $\left(\mathrm{E}^{\mathrm{q}} \varepsilon_{2}\right)^{1 / \mathrm{r}}$ and $\mathrm{E}$ is based on two preselected hypotheses of $q$ and $r$ together, an important aspect accentuates in the interpretation of optical experimental data for the high absorption region from the optical absorption edge of amorphous semiconductors, a different pair of selected parameters $q$ and $r$ can be chosen to obtain a good fitting with the straight line Eq. but with a different value of the optical energy gap for each pair of these parameters. Because of the double dependence of $\varepsilon_{2}$ on the parameters $\mathrm{q}$ and $\mathrm{r}$ in Eq. (1) inseparably, the above $\left(\mathrm{E}^{\mathrm{q}} \varepsilon_{2}\right)^{1 / \mathrm{r}}$ and $\mathrm{E}$ linear fitting is not a crucial measure to determine the reliable value of the optical energy gap, any appropriate pair of $q$ and $r$ values can be chosen to obtain a straight line and then a certain value of the optical energy gap, and because there are a large number of possible pairs of different values, there are also the same number of different optical energy gaps. The nature of this problem is illustrated by an experimental fact that there is more than one method in dealing with the optical data of a-Si:H, such as Tauc and Cody [9, 10]. Tauc put the first model to describe the optical transitions in a-semiconductors, he assumed that the fundamental difference with c-semiconductors is that the wave vector $(k)$ is not conserved, this is due to the change of the wave function property, some of which are localized over a specified region around each atomic site instead of being extended over the sample size as a whole as in the crystals [10]. He also assumed that the momentum matrix element is independent on photon energy and that the distribution of DOS for both the valence and conduction bands is in the form of parabola, at high values of photon energy around the assumed optical gap value, the equation of the imaginary dielectric function is given according to the Tauc model as follows:

$\mathrm{E} \varepsilon_{2}{ }^{1 / 2}=A\left[E-\mathrm{E}_{\mathrm{Opt}}\right]$

where $\mathrm{A}$ is a constant that contains the momentum matrix elements, $\mathrm{E}_{\mathrm{Opt}}$ is the optical energy gap which is called the Tauc gap and it is theoretically defined from the edges of both the valence and conduction bands. Thus, the optical energy gap of asemiconductor can be calculated by plotting $\mathrm{E} \varepsilon_{2}{ }^{1 / 2}$ as a function of photon energy $E$, this is named a Tauc plot. The optical gap is estimated by interpolation the straight line to the energy axis. There is also the Cody model, which assumes that the distribution of DOS near the valence and conduction bands edges is also parabolic and a non-dependence of a dipole matrix element on photon energy. The Eq. (3) of the imaginary dielectric function is given as follows: [11].

$\varepsilon_{2}{ }^{1 / 2}=B\left[\mathrm{E}-\mathrm{E}_{\mathrm{Opt}}\right]$ 
where $\mathrm{B}$ is a constant which contains a dipole matrix element and an $E_{O p t}$ optical energy gap which is called the Cody gap. Thus, the optical energy gap of amorphous conductor can be evaluated by plotting $\varepsilon_{2}{ }^{1 / 2}$ as a function of photon energy $E$, this is called the Cody plot. The optical gap is estimated by extrapolation of the straight line to the energy axis [8].

In fact, the Tauc relationship represented in Eq. (2) is not applied or observed in all a-semiconductors. It has been observed that there is a deviation from the dependence of the absorption coefficient on the photon energy square for some asemiconductors, i.e. there is linear dependence of the absorption coefficient on the photon energy $[13]\left[(E \alpha) \propto\left(E-E_{0}\right)\right]$ for amorphous selenium (a-Se) and also dependence of the absorption coefficient on the photon energy was found cubic [13] $\left[(\mathrm{E} \alpha)^{1 / 3} \propto\left(\mathrm{E}-\mathrm{E}_{0}\right)\right]$ for multicomponent chalcogenides. These deviations from the Tauc relationship was attributed because of the deviations of DOS functions from the simple power law. On the other hand, Singh suggested that $[13][\alpha(E) E]$ is dependent on the photon energy in the form of a polynomial of order 4, depending on which polynomial term may be most important in any material that can be obtained from the second, third or fourth degree of $[\alpha(E) E]$ on photon energy. In the latter case, Eq. (2) can be expressed as follows $[12,13]$ :

$\left[E^{2} \varepsilon_{2}(E)\right]^{x} \propto\left[E-E_{0}\right]$

where $x \leq 1 / 2$. Thus, any deviation from the square root $x=1 / 2$ or Tauc plot can be attributed to the photon energy-dependent momentum matrix element.

Singh's suggestion is useful, but is relatively impractical because of the limited flexibility of the polynomial function. It is better to assume that the dependence of the momentum matrix element on the photon energy through a continuous parameter $p$ as in the following suggested Equation:

$\left[E^{2} \varepsilon_{2}\right]^{\frac{1}{2}}=\mathrm{B}[\mathrm{E}]^{\mathrm{p}}\left[\mathrm{E}-\mathrm{E}_{0}\right]$

where the parameter $p$ represents the amount of systematic deviation of experimental data from the Tauc model when DOS is assumed to be a square root function. When the parameter $\mathrm{p}$ is equal to zero we have the Tauc model i.e. is a polynomial of the second order, when $\mathrm{p}$ equals one this is the case of the Cody model where the polynomial is of the fourth order. The difference from Singh's suggestion as what we assumed is that the deviation can be determined continuously from $p=0$ to $p=1$ or even outside these limits through the use of nonlinear regression method.

Levenberg-Marquadt method is used as a standard technique to solve the problems of non-linear least squares that arise when the fitting model function is nonlinear in the parameter [14]. The fitting function is of the following general form:

$\mathrm{y}=\mathrm{p}_{1} \mathrm{x}^{\mathrm{p}_{2}}\left(\mathrm{x}-\mathrm{p}_{3}\right)$

It is a function that contains three parameters for $p_{1}, p_{2}$ and $p_{3}$, in which are linear in the parameters $\mathrm{p}_{1}$, $\mathrm{p}_{3}$ and nonlinear in the parameter $\mathrm{p}_{2}$.

In addition to the method of nonlinear fitting, the derivative method is used as an independent method of data fitting. This method is expressed as follows [9, 15]:

$\left(\frac{\varepsilon_{2}}{\varepsilon_{2}}+\frac{\mathrm{q}}{\mathrm{E}}\right)^{-1}=\frac{\mathrm{E}-\mathrm{E}_{\mathrm{Opt}}}{\mathrm{r}}$

where $r$ and $q$ are defined in Eq. (1).

In the case of the assumption that the momentum matrix element is fixed, $\mathrm{q}=2$, in the case of the assumption that the dipole matrix element is fixed, $\mathrm{q}=0$ and $\mathrm{r}$ is a free parameter only if it 
is pre-selected, where $r=2$ is assumed in this research, the relation between the parameters $\mathrm{q}$ and $\mathrm{p}$ is $q=2-2 p$.

\section{Study of the experimental density of states of a-Si: $\mathbf{H}$}

Malik and O'Leary [16] re-analyzed DOS data for a-Si:H film prepared by Jackson et al. [17]. These two researchers used two methods to fit this data with the following Equation:

$\mathrm{N}_{c, v}=N_{c, v_{o}} \sqrt{\left|E-\mathrm{E}_{c, v}\right|}$

The first method selects a relatively large fitting range 1 in Fig. 1 and the second method selects a small fitting range 2 in Fig. 1, Malik and O'Leary showed that both modes of fitting conform well to the experimental data of Jackson et al. [16], but, from the observation of Fig. 1, the second method seems to more perfectly fitting the DOS data for both valence and conduction bands, while the first method is not as good as the two researchers claim. This is evident only by looking without further statistical analysis [16].

In order to enrich the physical meanings that can be derived from the data analysis of DOS above, the linear fitting results of Cody and Tauc for optical data $\varepsilon_{2}$ as a function of photon energy $\mathrm{E}$ can be resorted to. Jackson et al. found [16] that the Cody gap is $1.64 \mathrm{eV}$ for these films without indicating the fitting range, while O'Leary and Ibrahim [16, 18] found that the Cody gap is $1.68 \mathrm{eV}$ for the fitting range $(\sim 2-2.5 \mathrm{eV})$ and that the Tauc gap was determined by Jackson et al [16] as $1.86 \mathrm{eV}$. Ibrahim [6] also found that $J(E)$ proportional to the parabola Equation, Eq. (1).

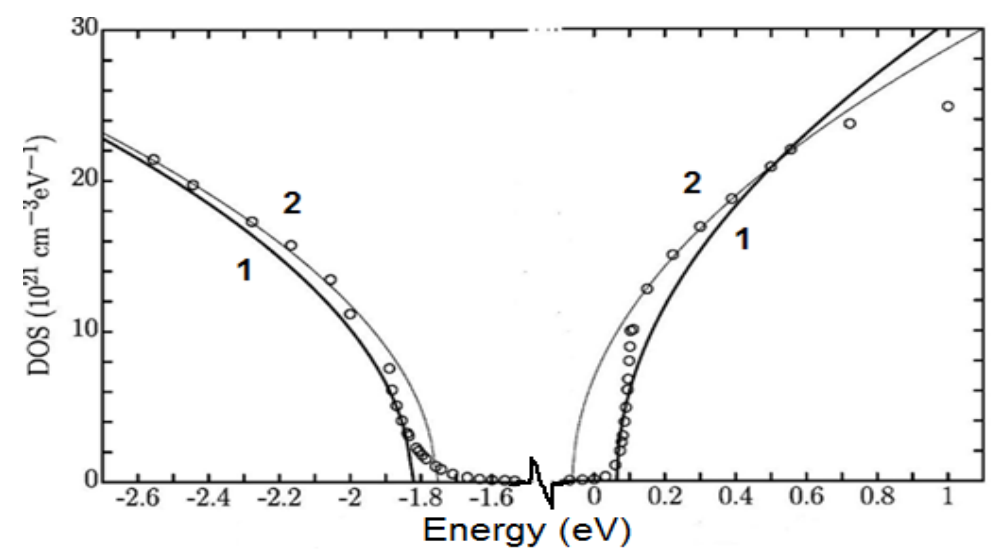

Fig. 1: Malik and $O^{\prime} L e a r y$ re-analysis DOS distribution data for a-Si: H films.

By benefiting from the results of the Malik and O'leary analysis [16] shown in Fig. 1, and by using the joint density of states function $\mathrm{J}(\mathrm{E})$ for a parabolic DOS function of the form

$J(E)=J_{0}\left(E-E_{\circ}\right)^{2}$

where $\mathrm{J}_{0}$ is the prefactor and $\mathrm{E}_{\mathrm{o}}$ is $\mathrm{E}_{\mathrm{c}_{\mathrm{o}}}-\mathrm{E}_{\mathrm{v}_{\mathrm{o}}}$ in Fig.2, it is found that,

(1) $\mathrm{J}_{\mathrm{o}}=3 * 10^{44} \mathrm{~cm}^{-6} \mathrm{eV}^{-3}$, and $\mathrm{E}_{\mathrm{c}_{\mathrm{o}}}-\mathrm{E}_{\mathrm{v}_{\mathrm{o}}}=1.89 \mathrm{eV}$ regarding the first method of fitting.
(2) $\mathrm{J}_{\mathrm{o}}=2.6 * 10^{44} \mathrm{~cm}^{-6} \mathrm{eV}^{-3}$, and $\mathrm{E}_{\mathrm{c}_{\mathrm{o}}}-\mathrm{E}_{\mathrm{o}}=1.7 \mathrm{eV}$ regarding the second method of fitting.

There are important conclusions regarding these results:

(a) The value of $\mathrm{J}_{0}$ for the second fitting is closer to the results obtained from the direct fitting of Jackson et al. $\mathrm{J}(\mathrm{E})$ results to Eq. (8) which is $2.25^{*} 10^{44} \mathrm{~cm}^{-6} \mathrm{eV}^{-3}$ [18], (b) The value of $E_{O p t}$ for the second fitting which is $1.7 \mathrm{eV}$ is very close to the 
value of the Cody gap for these films which is $1.68 \mathrm{eV}$ while the value of $\mathrm{E}_{\mathrm{Opt}}$ for the first fitting which is $1.89 \mathrm{eV}$ is closer to the Tauc gap value which is $1.86 \mathrm{eV}$.

In fact, these conclusions show us that the meaning of the optical energy gap derived from the optical data of a$\mathrm{Si}: \mathrm{H}$ films can be simply interpreted by samples prepared by Jackson et al. [14], as an extension of the extended DOS in both the conduction and valance bands to the energy axis within the mobility gap where $\mathrm{N}_{\mathrm{v}}(\mathrm{E})$ and $\mathrm{N}_{c}(\mathrm{E})$ are zero. Fig. 2 shows this simplified interpretation of the optical energy gap and one does not expect this simple interpretation to be valid in the case of highly disorder models such as low-quality a-Si:H films or amorphous compounds.

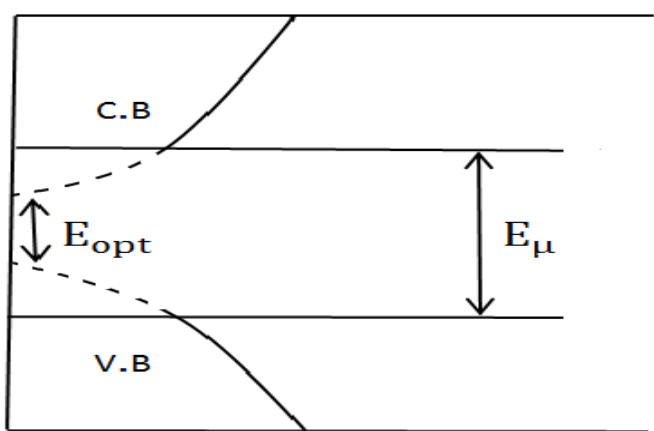

Fig. 2: The simplest interpretation of the meaning of the optical energy gap $E_{\text {opt }}$, where $E_{\mu}$ represents the mobility gap.

Then, the interpretation of the optical energy gap $E_{\text {opt }}$ obtained from the Cody plot of Jackson et al. films can be considered to be close to this simple interpretation of the optical energy gap. Based on our empirical analysis of DOS Fig.1, while the Tauc gap has no physical meaning to fit the practical results.

In fact, this simple change in the interpretation of the Cody gap proposed here has been used by Jiao et al. [19] for modeling purposes without empirical results. Here, experimental results show the possibility of this simple interpretation in the case of a$\mathrm{Si}: \mathrm{H}$ of Jackson et al. and it may be generalized to other high-quality films, where the effect of disorder is expected to be limited to the nature of the distribution of the extended DOS near two mobility edges of both the conduction and valance bands. In summary, it can be concluded that the a-Si:H films of Jackson et al. have the following characteristics:

(a) The distribution of the extended DOS near the mobility edges of the conduction and valance bands can be approximated from the parabola function.

(b) A square dipole matrix element is almost constant in a large range of photon energy.

(c) The notion added in this study is that the $E_{\text {opt }}$ can be physically interpreted as an extension of the extended DOS in both the conduction and valance band to the energy axis within the mobility gap where both $\mathrm{N}_{c}(\mathrm{E})$ and $\mathrm{N}_{\mathrm{v}}(\mathrm{E})$ are zero based on the analysis of empirical results of DOS and not theoretical modeling.

\section{Results and discussion}

Sample 1 (a-Si:H [17])

Films of a-Si:H were prepared by Jackson et al. in an ultrahigh-vacuum (UHV) method with pressure of $(0.5-2) \times 10^{-8}$ Torr all samples were deposited using 2-wrf power and $100 \%$ silane onto substrate held at $300^{\circ} \mathrm{C}$.

Fig. 3 shows a plot $\varepsilon_{2} / \varepsilon_{2}^{\prime}$ as a function of the photon energy of the a$\mathrm{Si}: \mathrm{H}$, it is observed from this Fig. that the data are well-fitting with the straight line Eq. expected from Cody plot results at using the derivative method $\varepsilon_{2} / \varepsilon^{\prime}{ }_{2}=(E-1.68) / 2$ in the fitting range $(\sim 2-2.7 \mathrm{eV})$. 


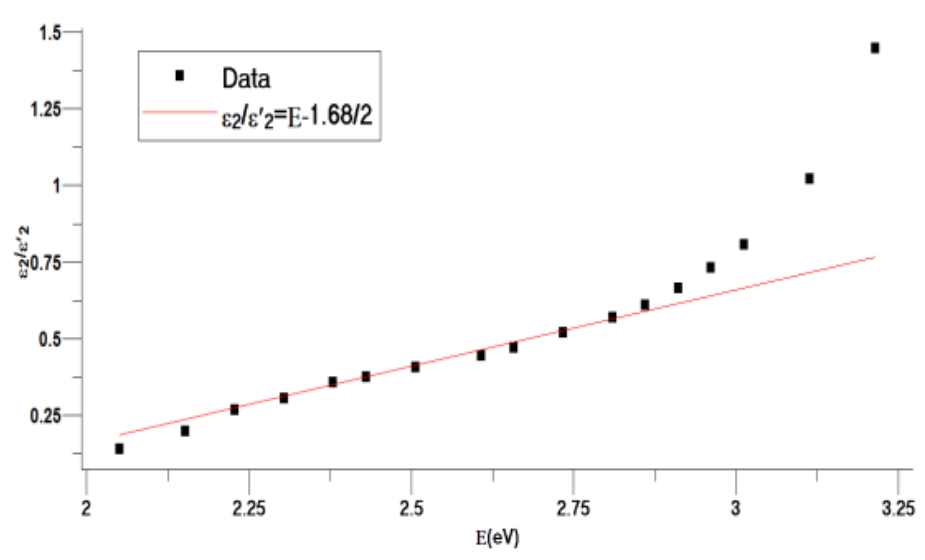

Fig. 3: $\varepsilon_{2} / \varepsilon^{\prime}{ }_{2}$ data for a-Si:H films.

Regarding the deviation from the straight line when $E>2.7 \mathrm{eV}$, it is explained by the decrease of the square of dipole matrix element with increasing of photon energy.

Fig. 4 shows the results of the $\mathrm{E} \varepsilon_{2}{ }^{1 / 2}$ plot as a function of the photon energy of a-Si:H samples in the fitting range $(\sim 2-2.7 \mathrm{eV})$ by using two methods of fitting, Fig. 4 (a) the Tauc plot, which considers the parameter $\mathrm{p}=0$, i.e., the fitting Eq. is a straight line Eq., Fig. 4 (b) the method which considers $\mathrm{p}$ is a free parameter for the purpose of assessing the deviation of the Tauc plot from the straight line Equation.

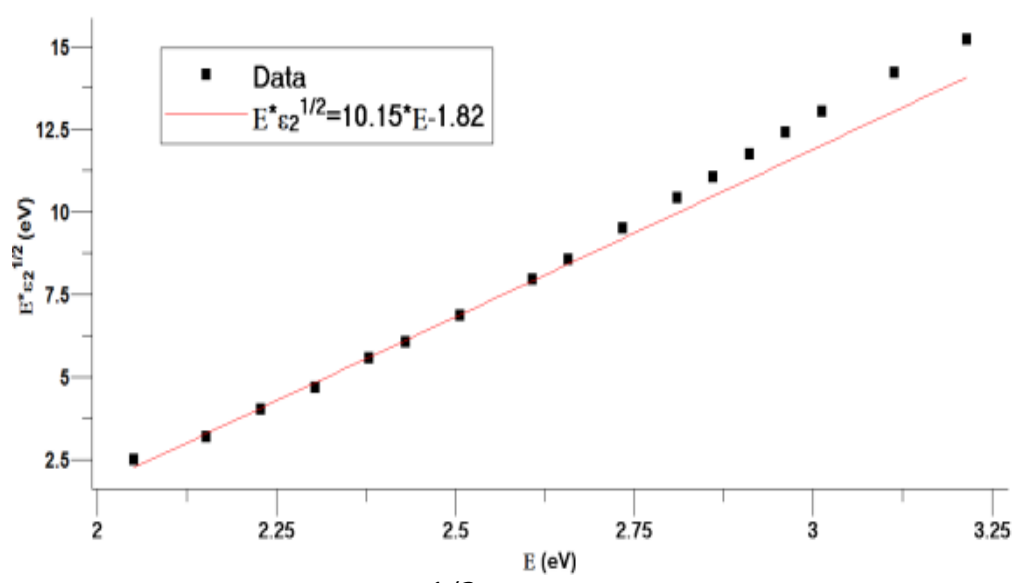

Fig. 4 (a): Data $E \varepsilon_{2}{ }^{1 / 2}$ for a-Si:H films when $p=0$.

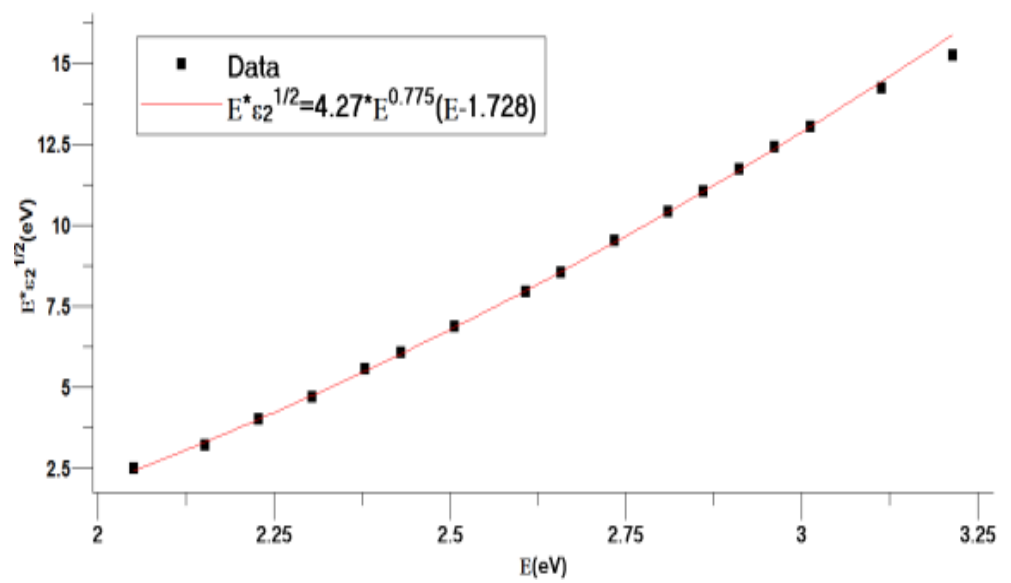

Fig. 4 (b): Data $E \varepsilon_{2}{ }^{1 / 2}$ for a-Si:H films where p is free. 
The selection of the fitting range is based on the fact that the matrix element begins to decrease slightly at the photon energy $(\sim 2-2.7 \mathrm{eV})$, which translates the observed increasing from $\varepsilon_{2} / \varepsilon^{\prime}{ }_{2}$ as a function of the photon energy Fig. 1. Table 1 shows the results of linear and nonlinear regression as well as Cody plot results in order to clearly show the deviation from Tauc plot.

Table 1: Linear and nonlinear regression results and Cody plot of a-Si: $\mathrm{H}$ films.

\begin{tabular}{|c|c|c|c|}
\hline Parameter & Tauc plot & Nonlinear plot & Cody plot \\
\hline $\mathrm{p}_{1}\left(\mathrm{~K}^{1 / 2}\right)$ & 10.15 & 4.27 & 3.35 \\
\hline $\mathrm{p}_{2}(\mathrm{P})$ & 0 & 0.775 & 1 \\
\hline $\mathrm{p}_{3}\left(\mathrm{E}_{\mathrm{opt}}\right)$ & 1.82 & 1.728 & 1.692 \\
\hline
\end{tabular}

It is noted that the value of parameter $\mathrm{p}_{2}$, which represents the parameter for the photon energydepending of the momentum matrix element, has a value of 0.775 for the fitting range $(\sim 2-2.7 \mathrm{eV})$ is closer to the $\mathrm{p}=1$ assumed by the Cody model. The optical energy gap obtained is $1.728 \mathrm{eV}$, although its difference from those obtained from Cody $1.68 \mathrm{eV}$ for the same range of fitting i.e., it is close to $1.7 \mathrm{eV}$ obtained from direct fitting with the DOS diagram (Fig. 1). It is observed from Tab. (1) that the values of the parameters obtained from the nonlinear regression method approach to the these obtained from the Cody plot for the same fitting range of the photon energy, with taking account for the expected error ratio at taking the data from the original plots by using the Get
Data program used here to obtain experimental points.

It is observed that the data do not take the shape of the straight line completely, but there is a slight but clear deviation which is observed arbitrarily and not quantitatively by the previous researchers [15] who made the Cody plot is a favorite for fitting in some of the literature of the optical properties of a-Si:H.

\section{Sample 2 (a-Si:H[20])}

Films of a-Si:H were prepared by Ferlauto et al. [20] by PECVD on glass substrates for $(0.5-1.5) \mu \mathrm{m}$ thicknesses. Fig. 5 shows the results of $\mathrm{E} \varepsilon_{2}{ }^{1 / 2}$ as a function of photon energy $E$ for these films in the fitting range $(2-2.75 \mathrm{eV})$ by using the nonlinear regression method.

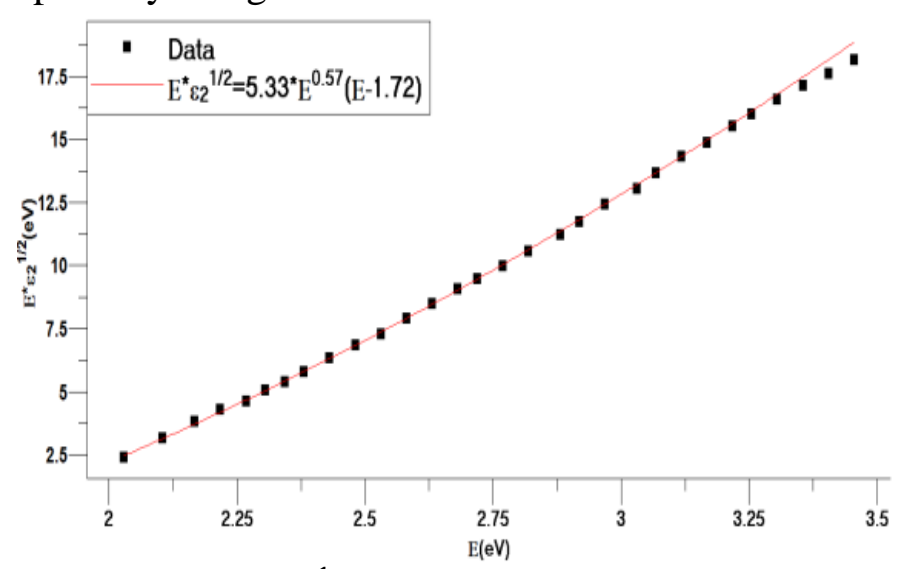

Fig. 5: Data $E \varepsilon 2^{1 / 2}$ for a-Si:H films, where $p$ is free. 
It is noted that the $\mathrm{p}_{2}$ value obtained here is 0.57 , which is far from the value $\mathrm{p}=0$ assumed by the Tauc model, and also from the $\mathrm{p}=1$ assumed by the model Cody. The value of parameter $\mathrm{p}_{2}$ is between Tauc and
Cody models. By using the derivative method. Table 2 shows the results of linear and nonlinear regression as well as Cody plot results in order to clearly show the deviation from Tauc plot.

Table 2: Linear and nonlinear regression results and Cody plot of a-Si:H films.

\begin{tabular}{|c|c|c|c|}
\hline Parameter & Tauc plot & Nonlinear plot & Cody plot \\
\hline $\mathrm{p}_{1}\left(\mathrm{~K}^{1 / 2}\right)$ & 10.17 & 5.33 & 3.25 \\
\hline $\mathrm{p}_{2}(\mathrm{P})$ & 0 & 0.57 & 1 \\
\hline $\mathrm{p}_{3}\left(\mathrm{E}_{\text {opt }}\right)$ & 1.80 & 1.72 & 1.63 \\
\hline
\end{tabular}

$\left(\frac{\varepsilon_{2}{ }^{\prime}}{\varepsilon_{2}}+\frac{\mathrm{q}}{2}\right)^{-1}$ as a function of the photon energy with assuming $\mathrm{q}=0$ (constant dipole matrix element) and $\mathrm{q}=2$ (constant momentum matrix element), Abeer found that the results in both cases are not fitting with both the Cody and Tauc plots [4], in addition to this is to find the parameter $p=1-q / 2$ from the nonlinear regression. In order to $\mathrm{o}$ test the probability of this value $\mathrm{p}$ in fitting with the optical data of Ferlauto et al., the derivative plot has been assumed $q=2-2 p=0.86$ where the value of $\mathrm{p}=0.57$.
Fig. 6 shows a relationship plot $\left(\frac{\varepsilon_{2}{ }^{\prime}}{\varepsilon_{2}}+\frac{0.86}{\mathrm{E}}\right)^{-1}$ as a function of photon energy, by fitting the data with the straight line Eq. $\mathrm{E}-\mathrm{E}_{\mathrm{Opt}} / 2$ by assuming that $r=2$ as also performed in this research - over the range of fitting $2-2.7 \mathrm{eV}$. The optical energy gap obtained was $1.71 \mathrm{eV}$ and corresponded to the value obtained by nonlinear regerission $1.72 \mathrm{eV}$, confirming that the appropriate $\mathrm{q}$ value of Ferlauto et al. is $q=2(1-p)=$ 0.86 .

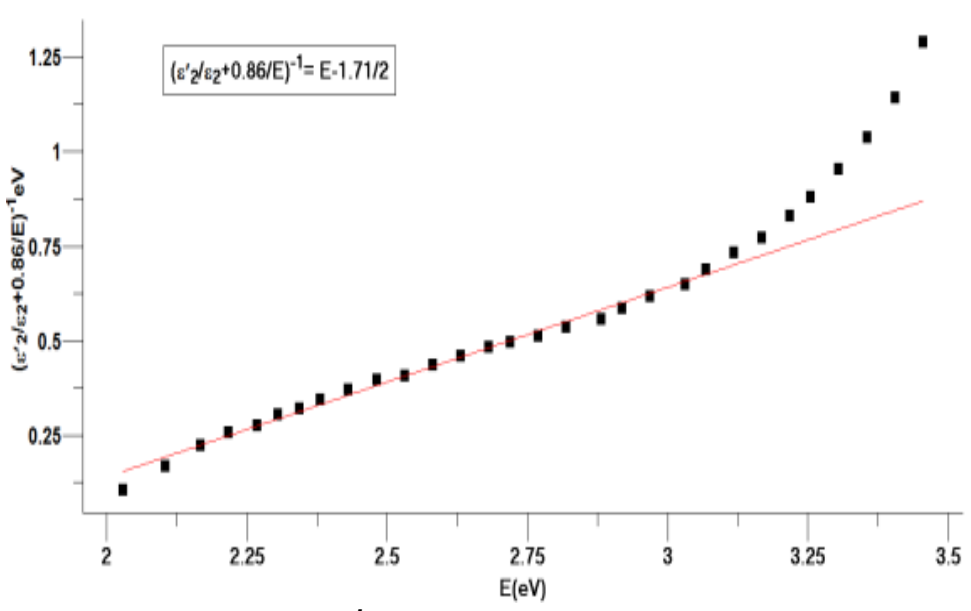

Fig. 6: Relationship plot $\left(\frac{\varepsilon_{2}{ }^{\prime}}{\varepsilon_{2}}+\frac{0.86}{E}\right)^{-1}$ as a function of the photon energy.

\section{Conclusions}

For a-Si:H films prepared by Jackson et al. the deviation parameter from Tauc plot is 0.787 i.e. the deviation is significant, but Ferlauto et al. a-Si:H films is the least deviation 
from the Tauc plot. The use of Cody plot is the most likely model which identifies with a-Si:H films overall, but there are exceptional cases where nonlinear regression is a more flexible and better method to obtain optical parameters.

\section{References}

[1] S. Nakhmanson, "Theoretical Studies of Amorphous and Paracrystalline Silicon", Ph.D. Thesis, the faculty of the College of Arts and Sciences of Ohio University. (2001).

[2] B. Akaglu, "Optical Properties of Silicon Based Amorphous Semiconductors Thin Films", Ph.D. Thesis, the Graduate School of Natural and Applied Sciences of Middle East. Technical University, (2004).

[3] S. Baranovski, edited, "Charge Transport in Disordered Solids with Applications in Electronics", John Wiley \& Sons Ltd. (2006).

[4] J. Singh, "Optical Properties of Condensed Matter and Applications", John Wiley \& Sons Ltd. (2006).

[5] S. Kugler and K. Shimakawa, "Amorphous Semiconductors", Cambridge University Press. (2015).

[6] R.A. Street, "Hydrogenated Amorphous Silicon", Cambridge University Press. (1991).

[7] J. I. Pankove, "Hydrogenated Amorphous Silicon", in: Willardson R.K. and Beer A.C. (Ed.), Semiconductors and Semimetals, 21D, Academic Press, New York. (1984).

[8] S. R. Elliott, "Physics of Amorphous Materials", Longman Group Limited. (1984).

[9] A. Abeer, "Theoretical Study of the High Absorption Region of the Optical Absorption Edge for Some Tetrahedral Amorphous Semiconductors using the derivative method", M.Sc. Thesis, University of Baghdad, (2014).
[10] J. Tauc, "Amorphous and Liquid Semiconductors", Plenum Publishing Company. (1974).

[11] G. D. Cody, "Hydrogenated Amorphous Silicon", in: Pankove J. I. (Ed.), Semiconductors and Semimetals, 21B, Academic Press, New York. (1984).

[12] M. Nessa, K. Shimakawa, A. Ganjoo, J. Singh, Optoelectron J. Adv. Mater., 2, 133 (2000) 5117-5128.

[13] J. Singh, Journal of Materials, (2003) 171-186.

[14] K. Madsen, N.B. Nielsen, O. Tingleff, "Methods for nonlinear least squares problems", Technical Report. Informatics and Mathematical Modeling, Technical University of Denmark. 2004.

[15] Farman K. Ahmed. "Theoretical Study of Optical Properties of Absorption Region of Amorphous Germanium and Silicon", MSc. Thesis, University of Baghdad, Iraq, (1996).

[16] Saad Malik and Stephen K. O'Leary, Journal of Materials Science, (2005) 177-181.

[17] W. B. Jackson, S. M. Kelso, C. C. Tsai, Allen W., Oh S.-J. Phys. Rev., (1985) 5187-5198.

[18] A. Ibrahim "A Theoretical Study of the High Absorption Region of the Optical Absorption Edge of Hydrogenated Amorphous Silicon", (in Arabic). Ph.D. Thesis, University of Baghdad, Iraq, (2002).

[19] L. Jiao, I. Chen, R.W. Collins, C.R. Wronski, N. Hata, Appl. Phys. Let., 72, 9 (1998) 1057-1059. [20] A. S. Ferlauto, G. M. Ferreira, J. M. Pearce, C. R. Wronski, R. W. Collinsa, J. Appl. Phys. 92, 5 (2002) 2424-2436. 